\title{
TEM Study of the Morphology of Nanoparticles
}

\author{
J. Deneen, P. Cernetti*, R. Gresback*, U. Kortshagen* and C.B. Carter
}

Department of Chemical Engineering and Materials Science, University of Minnesota, 421 Washington Ave SE, Minneapolis, MN 55455

* Department of Mechanical Engineering, University of Minnesota, 111 Church Street SE, Minneapolis, MN 55455

The use of nanoparticles in various applications requires consistent production of particles with uniform size and shape, especially since it has been widely shown that their properties can deviate from bulk behavior at this small scale $[1,2]$. Nanoparticles tend to differ from bulk material in part because their surface to volume ratio is much larger. Very small particles can have morphologies which differ from that of bulk material if this provides for lower-energy surfaces. Clearly, since the surface is so important for such small particles, it is crucial to know which surfaces are present in order to understand and model their properties.

Theoretical studies predict morphology changes as a function of particle size [3], and experimental evidence agrees. Small particles have been observed to take on specific geometric shapes such as $\mathrm{Si}$ cubes [4], Si and Ti spheres [5], and Au and FePt truncated octahedra [6, 7]. Simple morphologies can easily be deduced through traditional imaging and diffraction techniques in the transmission electron microscope (TEM). For more complex geometries tilting experiments can provide useful information about particle morphology, though there is considerable difficulty in tilting the nanoparticle while keeping it in the field of view.

The highly oriented germanium particles studied here were formed in a quartz-tube reactor using a glow capacitively coupled plasma. They were then extracted into a low-pressure chamber through a $1 \mathrm{~mm}$-diameter orifice and collected on an amorphous support-film for TEM analysis. When examined in the TEM a large fraction of the particles appeared to be hexagonal when viewed on the grid without tilting. In order to determine the true morphology of the particles and to identify the surface facets present, a single particle was tilted to three different zones (Fig. 1). In A the particle is oriented along a $<233>$ zone, in $\mathrm{B}$ it has been tilted to $\mathrm{a}<112>$ zone, and in $\mathrm{C}$ it has been tilted to a $<110>$ zone. All three orientations create a particle profile consistent with a slightly distorted cuboctohedron geometry, as illustrated in the schematic just next to each image.

The TEM is an indispensable tool for determining particle morphology and identifying surface facets as illustrated here for germanium nanoparticles. While electron tomography can also be used to create a three dimensional image of a particle [8], the diffraction information necessary to accurately determine the surface facets is only possible through systematic tilting. In this paper, reasons for the development of cuboctahedron and the deviations from perfect symmetry will be discussed.

References

[1] Buffat, P. and J.-P. Borel, Phys. Rev. A 13(6) (1976) 2287.

[2] Gerberich, W.W., et al., J. Mech. Phys. Solids, 51 (2003) 979. 
[3] C. Barreteau, et al., Eur. Phys. J. D 11 (2000) 395.

[4] Bapat, A, et al., J. Appl. Phys. 94(3) (2003) 1969.

[5] Perrey, C.R., et al, Microsc. Microanal. 10 Suppl.2 (2004) 55.

[6] Ascencio, J. A., et al. Surf. Sci. 447 (2000) 73.

[7] Dai, R.Z., et al., Surf. Sci. 505 (2002) 325.

[8] M. Weyland, et al., J. Phys. Chem. B 105 (2001) 7882.

[9] This work is funded through NSF grants NSF/DMI-0304211 and the IGERT-DGE-0114372.
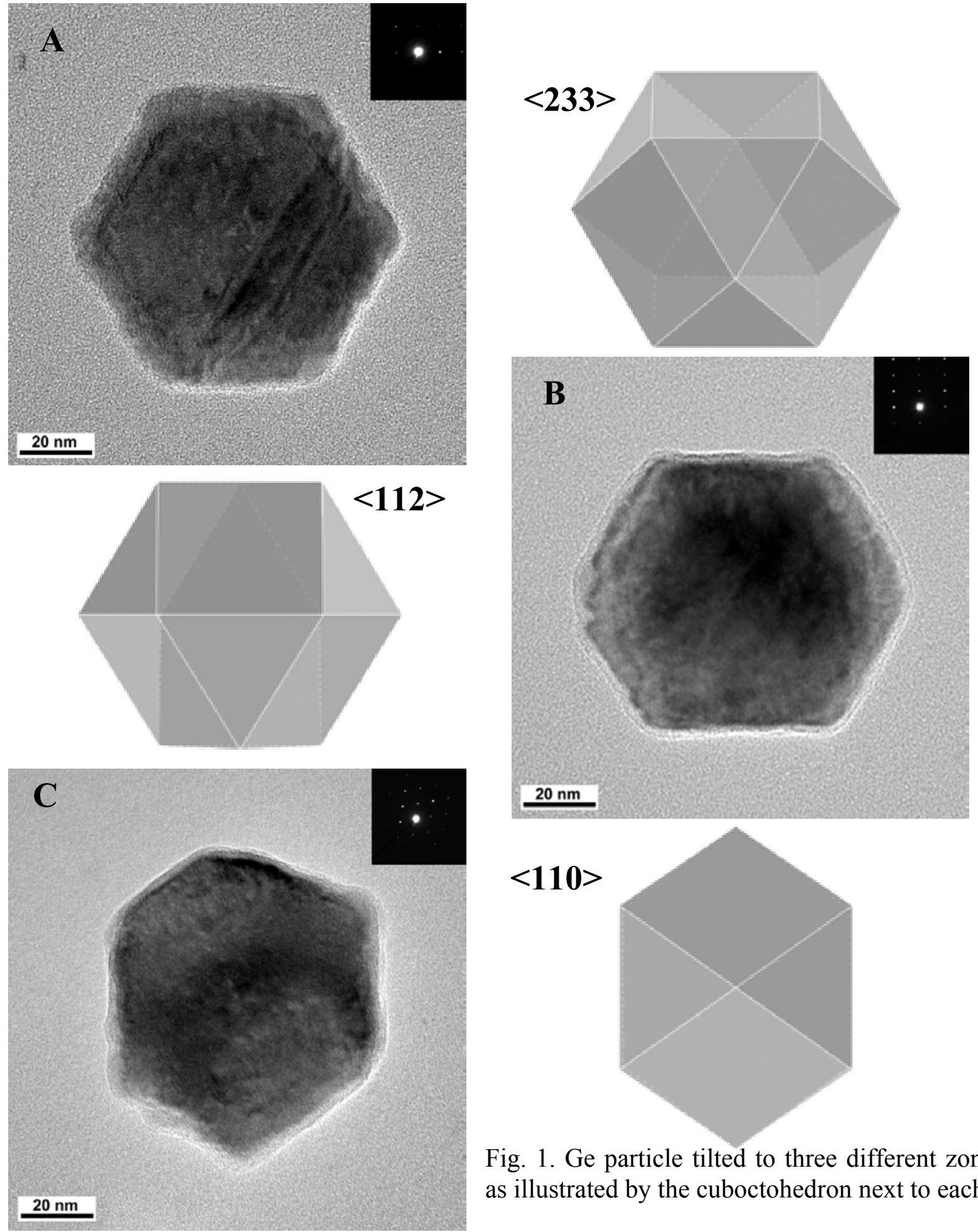

Fig. 1. Ge particle tilted to three different zones as illustrated by the cuboctohedron next to each. 